\title{
Catheter displacement prior to the delivery of high-dose-rate brachytherapy in the treatment of prostate cancer patients
}

\author{
Shogo Kawakami, MD!, Hiromichi Ishiyama, MD!, Tsuyoshi Terazaki, RT ${ }^{2}$, Itaru Soda, MD!, Takefumi Satoh, MD³, \\ Masashi Kitano, MD', Shinji Kurosaka, MD³, Akane Sekiguchi, MD', Shouko Komori, MD', Masatsugu Iwamura, MD³ \\ Kazushige Hayakawa, MD' \\ 'Departments of Radiology and Radiation Oncology, ${ }^{2}$ Division of Radiation Oncology, Kitasato University Hospital, Kitasato, Sagamihara, \\ ${ }^{3}$ Department of Urology, Kitasato University School of Medicine, Kitasato, Sagamihara, Japan
}

\begin{abstract}
Purpose: The purpose of this work was to report measured catheter displacement prior to the delivery of highdose-rate brachytherapy (HDR) in the treatment of prostate cancer.

Material and methods: Data from 30 prostate cancer patients treated with HDR brachytherapy were analyzed retrospectively. Eighteen transperineal hollow catheters were inserted under transrectal ultrasound guidance. Gold marker seeds were also placed transperineally into the base and apex of the prostate gland. Five treatment fractions of $7.5 \mathrm{~Gy}$ each were administered over 3 days. The patient underwent CT scanning prior to each treatment fraction. Catheter displacement was measured from the pre-treatment CT dataset reconstructed at $1.25 \mathrm{~mm}$ slice thickness.

Results: Most of catheters were displaced in the caudal direction. Variations of 18 catheters for each patient were small (standard deviations $<1 \mathrm{~mm}$ for all but one patient). Mean displacements relative to the apex marker were $6 \pm 4 \mathrm{~mm}, 12 \pm 6 \mathrm{~mm}, 12 \pm 6 \mathrm{~mm}, 12 \pm 6 \mathrm{~mm}$, and $12 \pm 6 \mathrm{~mm}$ from plan to $1^{\text {st }}, 2^{\text {nd }}, 3^{\text {rd }}, 4^{\text {th }}$, and $5^{\text {th }}$ fractions, respectively.

Conclusions: Our results indicate that catheter positions must be confirmed and if required, adjusted, prior to every treatment fraction for the precise treatment delivery of HDR brachytherapy, and to potentially reduce over-dosage to the bulbo-membranous urethra.
\end{abstract}

Key words: brachytherapy, catheter displacement, high-dose-rate, prostate cancer.

\section{Purpose}

Among the modern radiotherapeutic techniques, brachytherapy is an effective approach for delivering highdose radiation to a target. As a result, we have been treating prostate cancer patients with high-dose-rate (HDR) brachytherapy combined with hypofractionated external beam radiotherapy since 1999 [1,2]. The dosimetric accuracy of HDR brachytherapy relies on catheter positions being accurately reproduced to match the original positions in the planning CT dataset for all fractions of treatment. However, caudal catheter displacement is often observed prior to the first fraction [3,4] and between fractions [5-10]. This may lead to not only a significant risk of under-dosage at the target, but also over-dosage to the organs at risk. Several studies have demonstrated urethral stricture as the predominant late urinary toxicity of prostate HDR brachytherapy [2,11]. With significant caudal displacement, a high dose is likely to be erroneously delivered to the bulbo-membranous urethra. Sullivan et al. reported that $92 \%$ of all strictures occurred in the bulbo-membranous urethra, which lies distal to the prostatic apex [11]. Bulbo-membranous urethral stricture formation may, therefore, represent a clinical manifestation of caudal catheter displacement during HDR brachytherapy.

The purpose of this work was to determine catheter displacement prior to the delivery of HDR brachytherapy based on measurements from pre-treatment CT scan compared to the original planning CT scan with an implanted apex gold marker as representative of the bulbo-membranous urethra.

\section{Material and methods}

\section{Patients}

The institutional review board approved this retrospective study. Data from 30 prostate cancer patients treated

Address for correspondence: Hiromichi Ishiyama, MD, Department of Radiology and Radiation Oncology, Received: 3.12 .2013 Kitasato University School of Medicine, 1-15-1 Kitasato, Sagamihara, Japan, phone: +81-778-8453, Accepted: 13.05 .2014 fax: +81-778-9436, $₫$ e-mail: hishiyam@kitasato-u.ac.jp

Published: 26.06.2014 
Table 1. Patient characteristics

\begin{tabular}{lcc}
$n$ & & 30 \\
\hline Age & & $70(52-81)$ \\
\hline T stage & T1c & 6 \\
\cline { 2 - 3 } & T2b & 3 \\
\cline { 2 - 3 } & T2c & 4 \\
\cline { 2 - 3 } & T3a & 9 \\
\hline Initial PSA (ng/mL) & T3b & 8 \\
\hline Gleason score & & 13 \\
\cline { 2 - 3 } & 7 & 6 \\
\cline { 2 - 3 } & 8 & 3 \\
\hline Volume (ml) & 9 & 16 \\
\cline { 2 - 3 } & 10 & 12 \\
\cline { 2 - 3 } & $20-30$ & 2
\end{tabular}

Values are number or median (range)

with HDR brachytherapy were retrospectively analyzed. Patient characteristics are provided in Table 1.

\section{Catheter insertion and planning procedure}

Patients in the operating room were placed in a lithotomy position under epidural anesthesia. Multiple $24 \mathrm{~cm}$ long, closed-end, 6-F hollow plastic catheters were inserted transperineally using a Syed-Neblett plastic template (Alpha-Omega Services, Bellflower, CA, USA) under transrectal ultrasound guidance. Routinely, 18 catheters were implanted independently of prostate size. Twelve catheters were inserted in the peripheral portion, and six catheters were inserted in the central portion of the prostate. The needle tips were left within the urinary bladder $1.5 \mathrm{~cm}$ above the sonographically or cystoscopically defined base of the prostate. A CT scan was obtained for CT-based planning. The volumetric scans with reconstructed slice thickness of $1.25 \mathrm{~mm}$ were obtained using multi-detector row CT (Optima CT580, GE-Healthcare, WI, USA). The planning target volume (PTV) was defined as the prostate gland with or without proximal seminal vesicle, with a manually drawn margin between 3-5 $\mathrm{mm}$ in all directions. Reference points for the PTV were automatically distributed on the surface of the PTV. The dose limitation (maximum dose) was set as 8 Gy per fraction for the urethra, and 4 Gy per fraction for $5 \mathrm{~mm}$ behind the edge of the anterior rectal wall. A dose of 7.5 Gy per fraction to the PTV was prescribed, unless the dose limitation was violated, using inverse planning and geometric optimization. Five fractions of HDR treatment were administered. After CT-based planning performed on the Oncentra treatment planning system (Nucletron, Elekta $\mathrm{AB}$, Stockholm, Sweden), the first treatment session of HDR brachytherapy was conducted using the Nucletron microSelectron HDR ${ }^{192}$ Ir remote afterloading system. The first treatment session was conducted on the
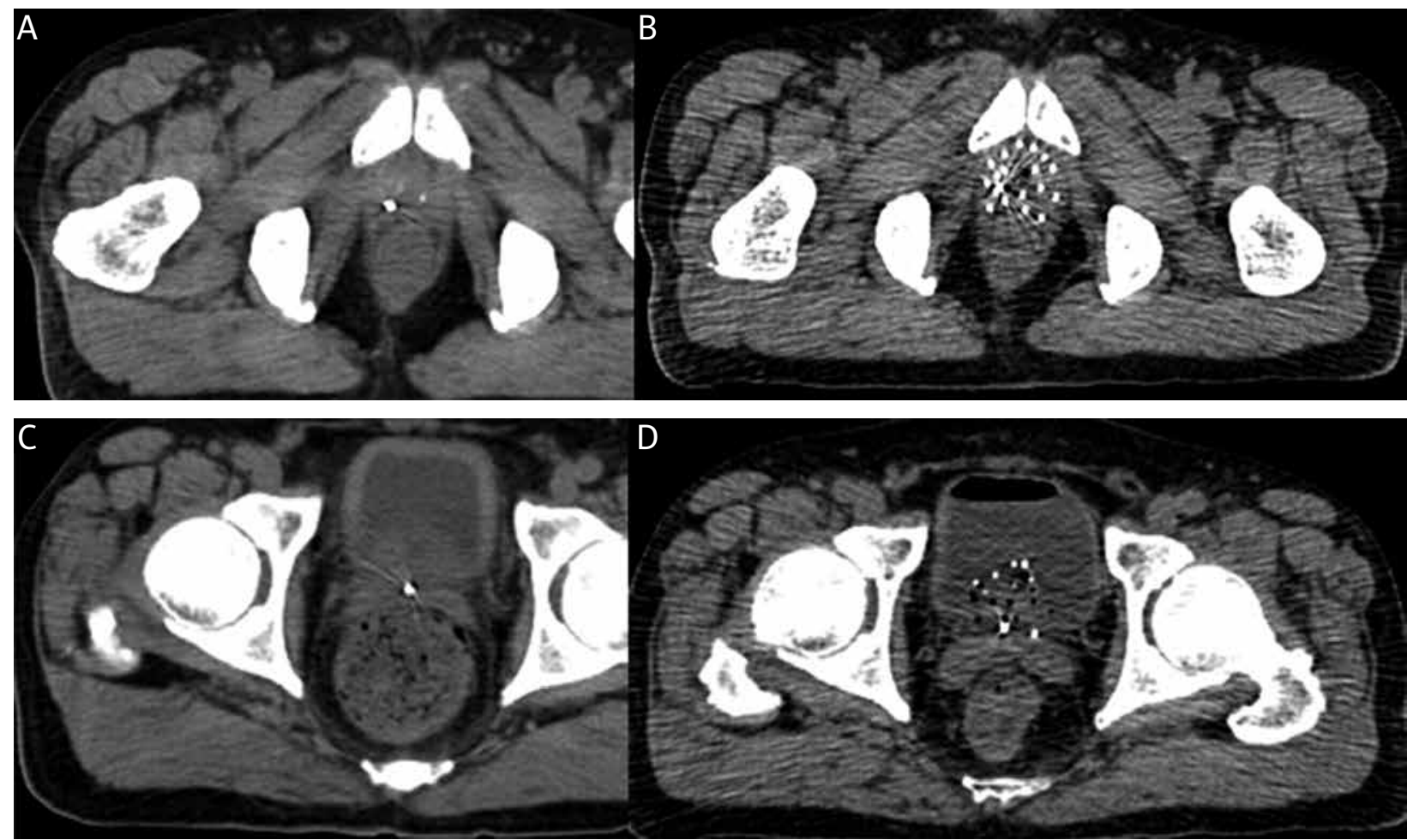

Fig. 1. Measurement of catheter displacement on CT images. Gold markers were implanted at the apex (A) and base (B) of the prostate gland. Catheter displacement was calculated by multiplying the thickness of the CT slice with the difference in number of CT slices between the slice of the apex gold marker $(C)$ and the reference slice detected by the pattern of the obturator markers (D) 


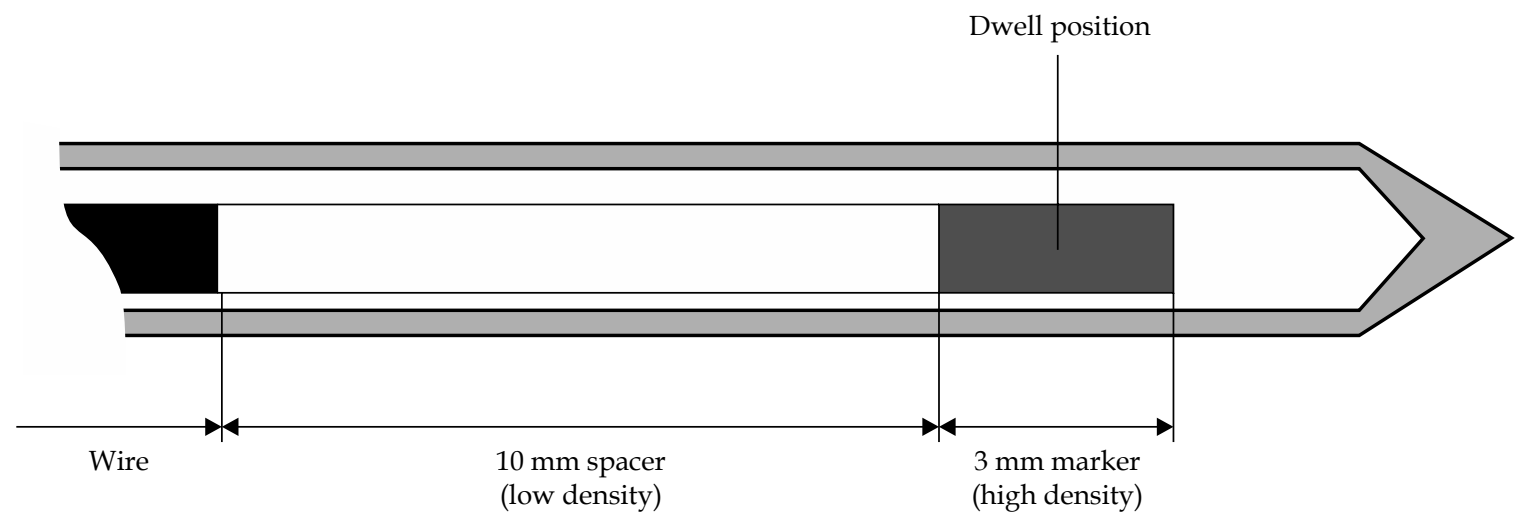

Fig. 2. Scheme of obturator

day of implantation, with the subsequent four treatment sessions administered twice daily on subsequent days. Basically, the time differences between plan CT and $1^{\text {st }}$, $2^{\text {nd }}, 3^{\text {rd }}, 4^{\text {th }}$, and $5^{\text {th }}$ fractions were $6 \mathrm{~h}, 24 \mathrm{~h}, 30 \mathrm{~h}, 48 \mathrm{~h}$, and $54 \mathrm{~h}$, respectively. Treatment duration of HDR was thus 3 days. Six days after completion of HDR brachytherapy, patients received EBRT using a dynamic-arc conformal technique, administered with high-energy photons comprising 10-MV X-rays (MHCL-15TP, Mitsubishi Electric, Tokyo, Japan) to a total dose of $30 \mathrm{~Gy}$. Total dose was administered in 5 weekly fraction doses of $3 \mathrm{~Gy}$. The radiation field was limited to the prostate gland with or without proximal seminal vesicles with a $7 \mathrm{~mm}$ leaf margin using multileaf collimators.

\section{Measurement of each catheter displacement between original planning scan and $1^{\text {st }}$ pre-treatment scan}

During catheter insertion, gold markers (VISICOIL; Iba-Dosimetry, Schwarzenbruck, Germany) were also implanted in the apex and base of the prostate. The apex-marker was used as a representative reference point for prostate and bulbo-membranous urethral position (Fig. 1A). Obturator with $3 \mathrm{~mm}$ marker and $10 \mathrm{~mm}$ spacer was inserted into every catheter (Fig. 2). A CT scan was acquired at $1.25 \mathrm{~mm}$ slice thickness prior to $1^{\text {st }}$ fraction in order to measure the catheters displacement relative to apex marker. The actual displacement was calculated by multiplying the thickness of the CT slice with the difference in number of CT slices between the slice of the apex marker and the marker of obturator of each catheter (Fig. 1D).

Catheter displacement $(\mathrm{mm})=(\mathrm{CT}$ slice number of obtulator marker - CT slice number of apex marker) $\times 1.25 \mathrm{~mm}$

The slice of an apex marker and an obturator maker were defined as the most cranial slices that showed the top of marker without artifact.

\section{Adjustment protocol}

In this study, measurements of each catheter position were done for only the first pre-treatment scan. Other catheter displacements were calculated by "slice-specific pattern" as a representative for all 18 catheter positions. Figure 3 shows our catheter adjustment protocol used in clinical practice. The obturators in 18 catheters make slice-specific patterns on CT-images (high and low density in the catheter hollows; Fig. 1D), because no catheter was inserted in completely equal depth, and that make it possible to recognize a representative slice for 18 catheters positions. Therefore, this slice-specific pattern on CT-image was used as a representative for all catheter position instead of measuring each 18 needles, because measuring all catheter positions in all treatments was not practical.

Where there was enough space between the first dwell position and distal end of the catheter hollow; dwell position was adjusted by changing indexer length at the treatment console. Where there was no space and displacement $>2 \mathrm{~mm}$, the catheter was manually advanced by

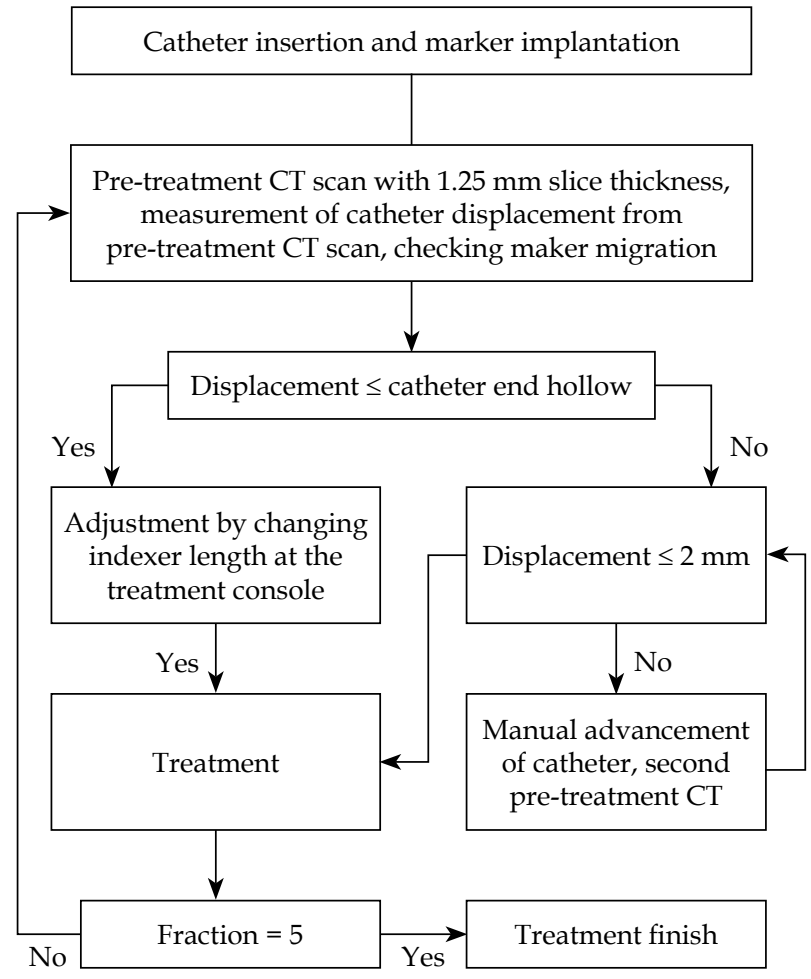

Fig. 3. Flow chart of the catheter adjustment protocol 
Radiation Oncologist. After manual advancement, a second CT was acquired to confirm the catheter position. The positions of gold markers implanted at the apex and base of the prostate were also checked by comparing with soft tissue anatomy on the CT images.

\section{Total catheter displacement in adjustment protocol}

The length of total catheter displacement was evaluated as follows:

$$
D_{\text {total }}=D_{\text {actual }}+D_{\text {manual }}
$$

where $\mathrm{D}_{\text {actual }}$ is actual displacement of the catheter calculated from difference between original planning CT and the first pre-treatment $\mathrm{CT}, \mathrm{D}_{\text {manual }}$ is actual length of manual catheter advancement calculated from difference between CTs scanned before and after manual advancement, and $\mathrm{D}_{\text {total }}$ is the sum of the two.

\section{Results}

\section{Catheter displacement}

In this manuscript, we used "mean \pm 1 standard deviation" for all our results. Table 2 shows actual displacements between original planning scan and $1^{\text {st }}$ pre-treatment CT scan of 18 needles for each patient. Standard deviations of 18 catheters for each patient (variation within one single patient) were $<1 \mathrm{~mm}$ except one. These small variations

Table 2. Displacements $(\mathrm{mm})$ between original planning CT and $1^{\text {st }}$ pre-treatment CT of 18 needles for each patient

Patients

Catheter places on template

Mean SD

\begin{tabular}{|c|c|c|c|c|c|c|c|c|c|c|c|c|c|c|c|c|c|c|c|c|}
\hline & 1 & 2 & 3 & 4 & 5 & 6 & 7 & 8 & 9 & 10 & 11 & 12 & 13 & 14 & 15 & 16 & 17 & 18 & & \\
\hline$A$ & 8 & 6 & 8 & 8 & 6 & 6 & 6 & 1 & 5 & 6 & 8 & 8 & 6 & 8 & 6 & 6 & 6 & 8 & 6 & 1 \\
\hline B & 3 & 3 & 3 & 3 & 1 & 3 & 1 & 3 & 1 & 3 & 3 & 4 & 3 & 3 & 1 & 3 & 3 & 3 & 2 & 1 \\
\hline$C$ & 0 & 0 & 1 & 3 & 1 & 1 & 3 & -1 & 1 & 1 & 3 & 4 & -1 & 0 & 0 & 1 & 3 & 0 & 1 & 1 \\
\hline $\mathrm{D}$ & 8 & 8 & 8 & 8 & 8 & 8 & 8 & 8 & 9 & 8 & 8 & 8 & 8 & 9 & 8 & 8 & 8 & 8 & 8 & 0 \\
\hline$E$ & 0 & 0 & 0 & 0 & 0 & 0 & 0 & -1 & 0 & 0 & 0 & 1 & 0 & 0 & 0 & 0 & 0 & 1 & 0 & 1 \\
\hline$F$ & 6 & 6 & 6 & 5 & 6 & 5 & 5 & 5 & 5 & 5 & 5 & 6 & 5 & 6 & 6 & 6 & 5 & 5 & 6 & 1 \\
\hline G & 9 & 8 & 8 & 6 & 6 & 6 & 6 & 5 & 6 & 6 & 6 & 8 & 8 & 6 & 6 & 6 & 6 & 8 & 7 & 1 \\
\hline $\mathrm{H}$ & 15 & 15 & 16 & 15 & 15 & 14 & 14 & 13 & 13 & 13 & 13 & 14 & 15 & 14 & 15 & 13 & 14 & 14 & 14 & 1 \\
\hline 1 & 11 & 11 & 10 & 9 & 9 & 9 & 10 & 9 & 9 & 9 & 10 & 11 & 10 & 10 & 9 & 9 & 10 & 10 & 10 & 1 \\
\hline J & 4 & 5 & 4 & 4 & 5 & 4 & 4 & 4 & 4 & 4 & 4 & 5 & 4 & 4 & 5 & 4 & 5 & 5 & 4 & 1 \\
\hline K & 8 & 9 & 10 & 9 & 8 & 10 & 10 & 10 & 10 & 9 & 10 & 9 & 9 & 9 & 9 & 9 & 9 & 10 & 9 & 1 \\
\hline $\mathrm{L}$ & 6 & 5 & 6 & 6 & 8 & 6 & 5 & 6 & 5 & 6 & 5 & 5 & 6 & 6 & 6 & 6 & 6 & 6 & 6 & 1 \\
\hline$M$ & 5 & 6 & 5 & 5 & 5 & 4 & 4 & 4 & 5 & 6 & 5 & 5 & 5 & 5 & 5 & 6 & 6 & 6 & 5 & 1 \\
\hline $\mathrm{NI}$ & 6 & 5 & 4 & 1 & 3 & 3 & NA & NA & 4 & 6 & 5 & 5 & 5 & 3 & 3 & 3 & 5 & 6 & 4 & 2 \\
\hline 0 & 14 & 14 & 15 & 15 & 14 & 15 & 14 & 14 & 14 & 14 & 14 & 14 & 14 & 14 & 14 & 14 & 14 & 14 & 14 & 0 \\
\hline$P$ & 5 & 5 & 5 & 5 & 5 & 5 & 5 & 5 & 6 & 6 & 5 & 5 & 5 & 5 & 6 & 6 & 5 & 5 & 5 & 1 \\
\hline Q & 4 & 4 & 4 & 4 & 5 & 5 & 4 & 4 & 4 & 3 & 3 & 4 & 5 & 4 & 4 & 4 & 4 & 4 & 4 & 1 \\
\hline$R$ & 8 & 9 & 8 & 8 & 6 & 6 & 6 & 6 & 5 & 5 & 8 & 8 & 8 & 6 & 5 & 6 & 6 & 6 & 7 & 1 \\
\hline$S$ & 4 & 4 & 4 & 4 & 4 & 4 & 4 & 5 & 5 & 5 & 4 & 4 & 3 & 4 & 4 & 5 & 5 & 4 & 4 & 1 \\
\hline $\mathrm{T}$ & 3 & 1 & 3 & 3 & 4 & 1 & 5 & 5 & 4 & 4 & 3 & 4 & 3 & 4 & 4 & 4 & 3 & 3 & 3 & 1 \\
\hline U & 6 & 8 & 6 & 6 & 8 & 6 & 6 & 6 & 5 & 5 & 5 & 6 & 6 & 6 & 6 & 6 & 6 & 6 & 6 & 1 \\
\hline V & 10 & 10 & 9 & 10 & 11 & 13 & 11 & 11 & 10 & 9 & 9 & 10 & 9 & 10 & 10 & 10 & 9 & 10 & 10 & 1 \\
\hline W & 0 & 0 & 0 & 0 & 0 & 0 & 0 & 0 & 0 & 0 & 1 & 0 & 0 & 0 & 0 & 0 & 0 & 0 & 0 & 0 \\
\hline$x$ & 14 & 14 & 15 & 14 & 14 & 14 & 13 & 13 & 13 & 14 & 14 & 14 & 14 & 13 & 13 & 13 & 14 & 14 & 13 & 1 \\
\hline Y & 10 & 10 & 11 & 11 & 11 & 11 & 10 & 10 & 11 & 11 & 11 & 10 & 11 & 10 & 11 & 11 & 11 & 11 & 11 & 1 \\
\hline Z & 3 & 3 & 3 & 3 & 4 & 3 & 3 & 1 & 4 & 3 & 3 & 4 & 3 & 3 & 3 & 4 & 3 & 3 & 3 & 1 \\
\hline AA & 13 & 13 & 14 & 14 & 15 & 14 & 14 & 14 & 13 & 13 & 14 & 13 & 13 & 14 & 14 & 13 & 14 & 11 & 13 & 1 \\
\hline BB & 9 & 9 & 9 & 9 & 9 & 9 & 9 & 10 & 9 & 9 & 10 & 9 & 10 & 9 & 9 & 10 & 10 & 8 & 9 & 1 \\
\hline CC & 10 & 9 & 9 & 8 & 8 & 8 & 6 & 8 & 8 & 9 & 9 & 10 & 8 & 8 & 6 & 8 & 9 & 9 & 8 & 1 \\
\hline $\mathrm{DD}$ & 11 & 11 & 11 & 10 & 11 & 10 & 10 & 9 & 9 & 9 & 10 & 11 & 10 & 10 & 10 & 9 & 10 & 10 & 10 & 1 \\
\hline
\end{tabular}

SD - standard deviation within each single patient 


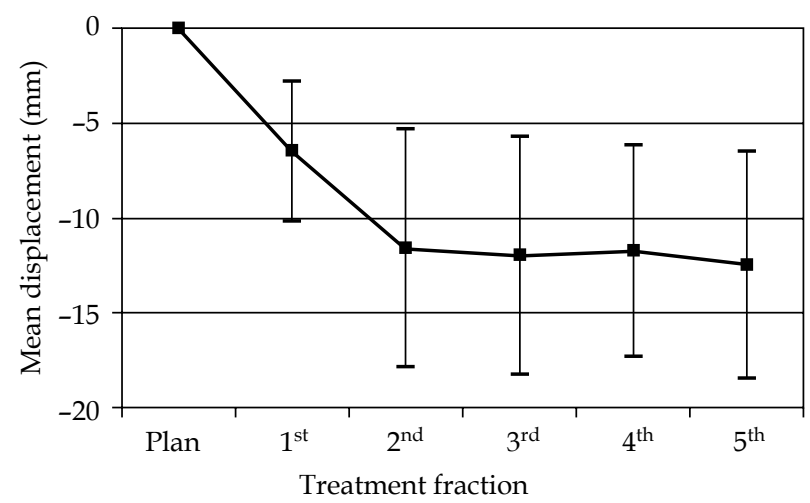

Fig. 4. Catheter displacement during 5 fractions of highdose-rate brachytherapy. Catheter displacements were seen especially in the $1^{\text {st }}$ and $2^{\text {nd }}$ fractions. Mean displacement was $6 \mathrm{~mm}$ between the plan and $1^{\text {st }}$, and $1^{\text {st }}$ and $2^{\text {nd }}$ fractions

among 18 catheters permit our "slice-specific pattern" recognition. Regarding total catheter displacement in the adjustment protocol, mean $D_{\text {total }}$ values were $6 \pm 4 \mathrm{~mm}$, $12 \pm 6 \mathrm{~mm}, 12 \pm 6 \mathrm{~mm}, 12 \pm 6 \mathrm{~mm}$, and $12 \pm 6 \mathrm{~mm}$ from plan to $1^{\text {st }}, 2^{\text {nd }}, 3^{\text {rd }}, 4^{\text {th }}$, and $5^{\text {th }}$ fractions, respectively. After the $2^{\text {nd }}$ fraction, catheter displacements were reduced (Fig. 4).

\section{Manual advancement of catheter}

Manual catheter adjustments were needed in 31 of 150 treatment fractions. Mean length of manual advancement was $10 \pm 3 \mathrm{~mm}$. After manual advancement, second pre-treatment CT was acquired (Fig. 3). On the second pre-treatment $\mathrm{CT}$, however, actual advancement was $4 \pm 3 \mathrm{~mm}$.

\section{Gold marker migration}

No displacement of apex fiducial markers compared to soft tissue anatomy was seen on CT images. Meanwhile, two patients displayed base-marker migrations of $20 \mathrm{~mm}$ and $25 \mathrm{~mm}$ just after implantation, and we failed to implant a base marker in 1 patient. In the remaining 27 patients, distances between apex and base markers gradually increased over time. Mean increases of distance between the two gold markers were $0 \pm 3 \mathrm{~mm}, 2 \pm 4 \mathrm{~mm}$, $3 \pm 4 \mathrm{~mm}, 4 \pm 2 \mathrm{~mm}$, and $3 \pm 4 \mathrm{~mm}$ from plan to $1^{\text {st }}, 2^{\text {nd }}$, $3^{\text {rd }}, 4^{\text {th }}$, and $5^{\text {th }}$ fractions, respectively.

\section{Discussion}

Because of the $1.25 \mathrm{~mm}$ CT slice thickness used, the lower limit of accuracy of our measurement was $1.25 \mathrm{~mm}$. As a result, our measurement data were written as sin-

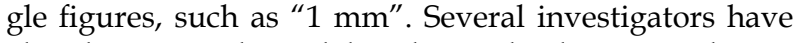
already reported caudal catheter displacement during HDR brachytherapy (Table 3) [3-7,9,10]. Although they used 3-5 mm CT slice thickness, mean displacements resembles our results were reported.

In our previous protocol, catheter displacements were checked by $\mathrm{X}$-ray films and adjusted if displacement was

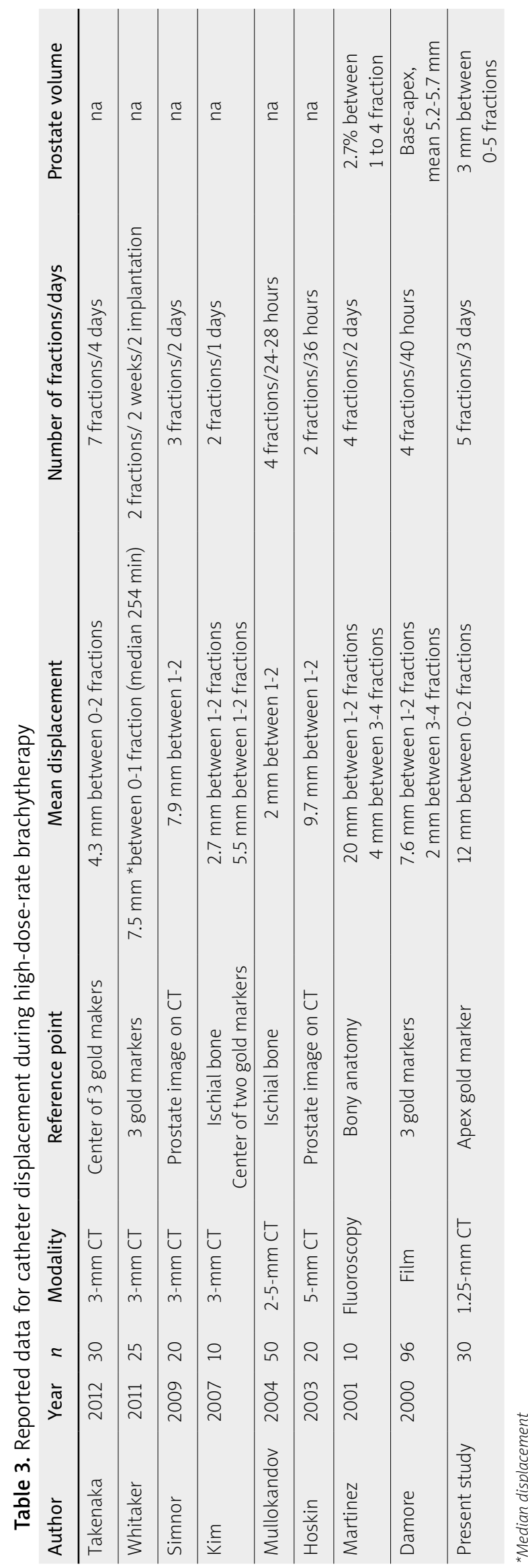

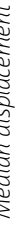


$>5 \mathrm{~mm}$ relative to implanted markers. However, $10 \%$ of our patients suffered from grade 3 genitourinary toxicity including urethral stricture [2]. Because caudal catheter displacement could be one of the reasons for urethral stricture [8,12], we changed our action level from $5 \mathrm{~mm}$ down to $2 \mathrm{~mm}$ with the aim of achieving a lower urethral dose. With this new protocol, exposure of the bulbo-membranous urethra to high-dose radiation could be avoided, and the urethral toxicity rate could be reduced. However, a longer follow-up time for the current patient cohort is required.

Our results also confirmed that the distances between apex and base markers were increased, suggesting that the prostate gland was gradually swelling throughout the course of treatment [13]. Herrmann et al. [14] also reported displacement of markers after HDR brachytherapy, especially in the superior-inferior direction (mean: $3 \mathrm{~mm}$ ). They discussed possible reasons for displacement such as localized bleeding into the prostate gland, or dislocation of prostate tissue inside the gland due to needle insertion. Although we added a 3- to 5-mm margin around the prostate, some parts of the PTV may not have received sufficient dose in some cases. Re-planning may be needed for patients with an excessively enlarged prostate after catheter insertion. Further investigation is required to resolve this problem.

On the other hand, our results showed no significant displacement of apex markers. The apex is, in comparison to other structures around the prostate, less dependent on internal influences such as swelling of the prostate, therefore it may represent a suitable position for marker implantation compared to the base of the gland.

Tiong et al. [15] examined the impact of catheter displacement on tumor control probability (TCP) in patients with prostate cancer receiving HDR, and advised that action levels to correct for catheter displacements should be $\leq 3 \mathrm{~mm}$. According to their calculations, median relative TCP was 0.998 , if catheter displacement was $3 \mathrm{~mm}$. We therefore set our catheter displacement action level at $2 \mathrm{~mm}$ (Fig. 3) to include a safety margin.

Another interesting point we found in this study was the difficulty of manually adjusting the catheter. Physically advancing the catheter $1 \mathrm{~cm}$ into the patient resulted in an actual advance of only approximately $0.4 \mathrm{~cm}$ relative to the apex marker, as the prostate itself was also pushed along with the catheter. This result suggests that a combination with computerized adjustment of dwell position in software is needed when manual catheter advancement is performed.

\section{Conclusions}

In conclusion, frequent catheter displacements relative to the apex of the prostate and bulbo-membranous urethra were confirmed by measurement prior to each treatment fraction on pre-treatment CT images with $1.25 \mathrm{~mm}$ slice thickness. Our results indicate that catheter positions must be confirmed and if required, adjusted, prior to every treatment fraction for the precise treatment delivery of HDR brachytherapy, and to potentially reduce over-dosage to the bulbo-membranous urethra.

\section{Disclosure}

This work was supported by JSPS KAKENHI Grant Number 24791334. Authors report no conflict of interest.

\section{References}

1. Egawa S, Shimura S, Irie A et al. Toxicity and health-related quality of life during and after high dose rate brachytherapy followed by external beam radiotherapy for prostate cancer. Jpn J Clin Oncol 2001; 31: 541-547.

2. Ishiyama $\mathrm{H}$, Kitano $\mathrm{M}$, Satoh $\mathrm{T}$ et al. Genitourinary toxicity after high-dose-rate (HDR) brachytherapy combined with hypofractionated external beam radiotherapy for localized prostate cancer: an analysis to determine the correlation between dose-volume histogram parameters in HDR brachytherapy and severity of toxicity. Int J Radiat Oncol Biol Phys 2009; 75: 23-28.

3. Takenaka T, Yoshida K, Ueda M et al. Assessment of daily needle applicator displacement during high-dose-rate interstitial brachytherapy for prostate cancer using daily ct examinations. J Radiat Res 2012; 53: 469-474.

4. Whitaker M, Hruby G, Lovett A et al. Prostate HDR brachytherapy catheter displacement between planning and treatment delivery. Radiother Oncol 2011; 101: 490-494.

5. Simnor T, Li S, Lowe G et al. Justification for inter-fraction correction of catheter movement in fractionated high doserate brachytherapy treatment of prostate cancer. Radiother Oncol 2009; 93: 253-258.

6. Kim Y, Hsu IC, Pouliot J. Measurement of craniocaudal catheter displacement between fractions in computed tomography-based high dose rate brachytherapy of prostate cancer. J Appl Clin Med Phys 2007; 8: 2415.

7. Mullokandov E, Gejerman G. Analysis of serial CT scans to assess template and catheter movement in prostate HDR brachytherapy. Int J Radiat Oncol Biol Phys 2004; 58: 1063-1071.

8. Hoskin PJ, Bownes PJ, Ostler P et al. High dose rate afterloading brachytherapy for prostate cancer: catheter and gland movement between fractions. Radiother Oncol 2003; 68: 285288.

9. Martinez AA, Pataki I, Edmundson G et al. Phase II prospective study of the use of conformal high-dose-rate brachytherapy as monotherapy for the treatment of favorable stage prostate cancer: a feasibility report. Int J Radiat Oncol Biol Phys 2001; 49: 61-69.

10. Damore SJ, Syed AM, Puthawala AA et al. Needle displacement during hdr brachytherapy in the treatment of prostate cancer. Int J Radiat Oncol Biol Phys 2000; 46: 1205-1211.

11. Sullivan L, Williams SG, Tai KH et al. Urethral stricture following high dose rate brachytherapy for prostate cancer. Radiother Oncol 2009; 91: 232-236.

12. Kolkman-Deurloo IK, Roos MA, Aluwini S. HDR monotherapy for prostate cancer: A simulation study to determine the effect of catheter displacement on target coverage and normal tissue irradiation. Radiother Oncol 2011; 98: 192-197.

13. Tejwani A, Bieniek E, Puckett $\mathrm{L}$ et al. Case series analysis of post-brachytherapy prostate edema and its relevance to post-implant dosimetry. Post-implant prostate edema and dosimetry. J Contemp Brachytherapy 2012; 4: 75-80.

14. Herrmann MK, Kertesz T, Gsänger T et al. Gold marker displacement due to needle insertion during HDR brachytherapy for treatment of prostate cancer: a prospective cone beam computed tomography and kilovoltage on-board imaging (kV-OBI) study. Radiat Oncol 2012; 7: 24.

15. Tiong A, Bydder S, Ebert $M$ et al. A small tolerance for catheter displacement in high-dose rate prostate brachytherapy is necessary and feasible. Int J Radiat Oncol Biol Phys 2010; 76: 1066-1072. 